\title{
Contact Angle Measurement at Aluminum Surfaces
}

\author{
B.A. Shenoi, S. Ramajayam and N. Rangarajan
}

Central Electrochemical Research Institute, Karaikudi, India

\begin{abstract}
The theory of contact angles of Doss and Rao has been extended to solid-liquid-liquid systems. The effect of roughness on the contact angles is discussed. The theory is used to interpret the wetting properties of aluminum surfaces subject to different methods of treatment.
\end{abstract}

\section{Introduction}

It is a well-known fact that the contact angle of liquid-solid interfaces is a very important criterion for the study of the wetting properties of solid surfaces. Many attempts have been made to measure the wetting property of solid surfaces by means of contact angle values. Richards and Carver ${ }^{1)}$ have reported contact angle values for solid-liquid-air interfaces by photographing the liquid meniscus in contact with the solid. Adam and Jessop ${ }^{2)}$ measured contact angle by the tilting plate method. Equilibrium contact angles were calculated by them from the values of advancing and receding contact angles. The spheroidal segment method reported dy G.L.Mack ${ }^{3)}$ has been adopted by us in the present investigation carried out with a view to study the preferential wetting properties of aluminum surfaces subject to different methods of treatment against liquid paraffin in the water phase. The theory of contact angles of Doss and $\mathrm{Rao}^{5,6)}$ has been extended to solid-liquid-liquid systems.

\section{Experimental}

The experimental set-up consists of a rectangular glass cell $\left(3^{\prime \prime} \times 0.5^{\prime \prime} \times 2.5^{\prime \prime}\right)$ with optically plane glass sides mounted on an adjustable platform. The metal plate to be tested is rested on the glass cell $G$, and adjusted for horizontality before the experiment. A small bent-pipette $\mathrm{P}$ is used for releasing the oil drops*. The drops are allowed to detach from the orifice "naturally" so that they have more or less the same size. The small cell is placed inside another glass cell $G^{\prime}$ covered by a Perspex sheet $C$, with provision for keeping the bent-pipette. An optical arrangement is made for illuminating the drop. The drop is viewed through a travelling microscope $M$, having horizontal and vertical movements.

\section{Pretreatment of the metal surface}

(i) Polished aluminum surfaces : Superpurity aluminum (99.99\%) strips of the size $2^{1} / 2^{\prime \prime} \times 1 / 2^{\prime \prime}$ $\times 1 / 32^{\prime \prime}$ are mechanically polished to a mirror finish taking care to avoid hills and valleys on the metal surface. After polishing, the strips are dipped in a mixture of phosphoric acid and chromic acid at

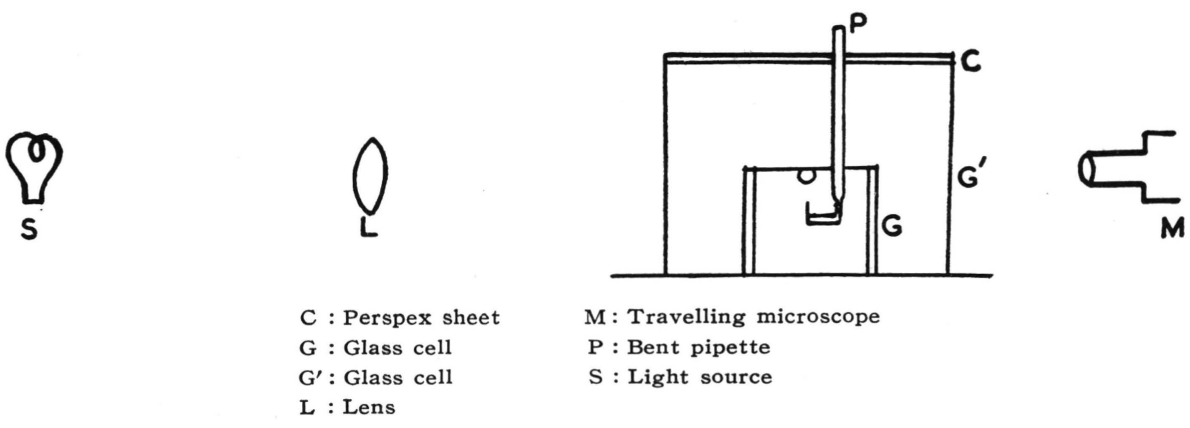

Fig. 1 Experimental set-up

\footnotetext{
* The details of the pipette were kindly furnished by Dr. W.H. Banks.
} 
$90^{\circ}-100^{\circ} \mathrm{C}$ for removing the adsorbed impurities. Then they are washed in running tap water, distilled water and dried, vapour degreased in benzene for one hour, heated in air at $100^{\circ} \mathrm{C}$ and allowed to cool slowly for one hour. $\left.{ }^{4}\right)$

(ii) Treatment for anodized plates : The pretreatment is the same as for polished surfaces. But after vapour degreasing the plate is introduced into the desired anodizing bath and the anodization carried out under the specified conditions. After anodizing, the aluminum plate is washed in running tap water and distilled water. The plate is used without sealing in the experiment.

(iii) Treatment for etching aluminum: After vapour degreasing, the plate is etched in $20 \%$ $\mathrm{HCI}+0.1 \% \mathrm{CuCl}_{2}$ at room temperature for two minutes. After etching, the plate is washed in $5 \% \mathrm{HNO}_{3}$, then in running tap water and distilled water.

\section{Measurement of contact angle}

After the particular treatment is over, the aluminum plate is immediately transferred to the glass cell which has previously been cleaned, and filled to the brim with distilled water, sweeping the water surface free of contamination with a paraffined glass strip. After fifteen minutes the liquid paraffin drop is released to the metal plate. The drop is allowed to grow gradually and detach spontaneously. Measurements of the dimensions, $d$ and $h$, of the oil drop were made after about fifteen minutes. $\theta$ is calculated from the values of $d$ and $h$, where $d$ is the diameter of the base of the liquid segment and $h$ is the maximum height of the drop.

$$
\cos \theta_{32}=\frac{h^{2}-(d / 2)^{2}}{h^{2}+(d / 2)^{2}}
$$

$\theta_{32}$ is the average contact angle occurring along the perimeter of the drop. The subscript 1 stands for solid phase, 2 stands for aqueous phase and 3 stands for non-aqueous phase. The interfaces are represented by subscripts 13,12 and $32 . \theta_{32}$ represents the angle made by the organic liquid-water interface 32 with the solid and measured from the interface towards the solid in the direction 32 . For acute angles $\cos \theta_{32}$ is positive and for obtuse angles $\cos \theta_{32}$ is negative. A rise in contact angle indicates a lowering of hydrophilic property of the metal surface (vide infra).

Data

The following table gives the contact angle values for the aluminum plates treated under different conditions :

Table 1 Values of Pore Diameter for the Plates Anodized in Different Electrolytes

\begin{tabular}{|c|c|c|c|c|}
\hline S. No. & & System & & $\theta_{32}$ \\
\hline 1 & Mechanically polished & Liquid paraffin & Distilled water & $74^{\circ}$ \\
\hline 2 & $"$ & Oleic acid & $"$ & $120^{\circ}$ \\
\hline 3 & $"$ & Liquid paraffin $+5 \%$ Oleic acid & " & $130^{\circ}$ \\
\hline 4 & Electropolished & $\Rightarrow$ & $"$ & $98^{\circ}$ \\
\hline 5 & Anodized (in $\mathrm{H}_{2} \mathrm{SO}_{4}$ bath) & $"$ & $"$ & $54^{\circ}$ \\
\hline 6 & Anodized (in $\mathrm{H}_{2} \mathrm{Cr}_{2} \mathrm{O}_{7}$ bath) & $"$ & $"$ & $39^{\circ}$ \\
\hline 7 & Anodized (in $\mathrm{H}_{3} \mathrm{PO}_{4}$ bath) & $"$ & $"$ & $27^{\circ}$ \\
\hline 8 & Etched & $"$ & $"$ & $39^{\circ}$ \\
\hline
\end{tabular}

\section{Conclusions}

(i) Polished (mechanically as well as electrolytically) aluminum plates give different values of $\theta_{32}$ though the treatment is closely followed in each case. But the values are all above $90^{\circ}$. With liquid paraffin alone $\theta_{32}$ is lower for polished surface. But if a little oleic acid is added to liquid paraffin $\theta_{32}$ is increased.

(ii) Electropolished aluminum surfaces show lower contact angle than mechanically polished surfaces.

(iii) Anodized aluminum plates differ in their wetting properties profoundly from polished surfaces. While the polished surfaces are difficultly wetted by water, anodized plates have $\theta_{32}<90^{\circ}$. Values 
of $\theta_{32}$ vary with plates anodized in different baths.

(iv) Etched aluminum plate behaves like anodized plate.

\section{Discussion}

\section{Theory of Contact Angles}

Doss and $\mathrm{Rao}^{5,6)}$ have developed a theory of contact angles for the case of a solid-liquid-vapour system. Livingston ${ }^{7)}$ has examined the then available data and has concluded that all the available data are in agreement with the theory. This theory has been extended to the case of the solid-liquid-liquid system in this paper.

For the solid-liquid-liquid system, the Young's equation ${ }^{8)}$ can be written as

$$
S_{13}-S_{12}=S_{32} \cos \theta_{32}
$$

where $S_{13}=$ Interfacial tension of the solid-organic liquid interface;

$S_{12}=$ Interfacial tension of the solid-water interface;

$S_{32}=$ Interfacial tension of the organic liquid-water interface.

Under equilibrium conditions ${ }^{9}$, the above equation can be written as

$$
S_{13}^{\prime}-S_{12}^{\prime}=S_{32} \cos \theta_{32}
$$

where $S^{\prime}{ }_{13}$ is the free energy per unit area of the solid in contact with the organic liquid saturated with water, and $S^{\prime}{ }_{12}$ the free energy per unit area of the solid in contact with water saturated with the organic liquid.

The relation between preferential adsorption and contact angle can be derived as follows :

Consider the solid immersed in an organic liquid saturated with water. There is preferential adsorption of one of the liquids and the solid surface would be partially or completely covered with an adsorption layer of water, depending upon the nature of the substances involved. If the principle of independent surface action of Langmuir ${ }^{10}$ is applied to this system, the solid-organic/liquid interface then really consists of three interfaces, the solid-organic/liquid, the organic/liquid-water and the solidwater. Let a fraction $\sigma$ of the total surface of the solid be covered by the water molecules (Fig. 2(a)).

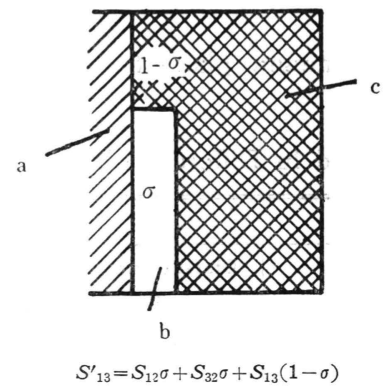

a : Solid (1)

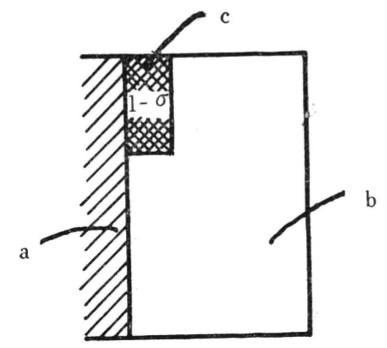

$S_{12}=S_{12} \sigma+S_{13}(1-\sigma)+S_{32}(1-\sigma)$
Fig. 2 (a) Plate dipped in organic liquid saturated with water

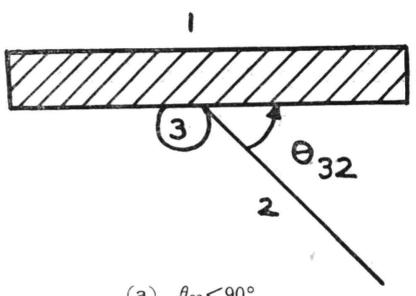

(a) $\theta_{32}<90^{\circ}$ b : Water (2)
Fig. 2 (b) Plate dipped in water saturated

c : Organic liquid (3) with organic liquid



(b) $\theta_{32}>90^{\circ}$

1 : Solid phase 2: Aqueous phase $3:$ Non-aqueous phase

Fig. 3 (a) and (b) Schematic diagram defining $\theta_{32}$ when (a) acute and (b) obtuse 
The actual solid-water interface per unit area of the solid surface as pictured, would be $\sigma$. Hence the solid-organic/liquid interface would be $(1-\sigma)$. The specific free energy of 'apparent solid-liquid interface' is given by the equation

$$
S_{13}^{\prime}=S_{12} \sigma+S_{32} \sigma+S_{13}(1-\sigma)
$$

Here $S_{12}$ represents the interfacial energy of the solid-water interface per unit area of the actual contact between the solid and water.

If the solid is now immersed in water, we can assume that there is no alteration in the fraction of the surface of the solid covered by water molecules since the solid has already been in equilibrium with water. The specific free energy of 'apparent solid-water interface' can be written as follows (Fig. 2 (b)) :

$$
S^{\prime}{ }_{12}=S_{12} \sigma+S_{13}(1-\sigma)+S_{32}(1-\sigma)
$$

From equation (2), (3) and (4) we get

$$
\cos \theta_{32}=(2 \sigma-1)
$$

If $\sigma=1, \theta_{32}=0$. This represents complete wetting of the solid by water. If $\sigma=1 / 2, \theta_{32}=\pi / 2$. This corresponds to the equal wetting of the solid by water and the organic liquid. If $\sigma=0, \theta_{32}=\pi$, which corresponds to the case of complete wetting by the organic liquid, and absence of wetting by water.

The influence of roughness may be considered next. A rough surface consists of craters which form a fraction of the solid surface.

(i) If $\theta_{32}$ is acute, the craters are completely covered with water. Then

$S^{\prime}{ }_{13}=$ Solid-organic/liquid interfacial energy $+S_{32}\{x+\sigma(1-x)\}+$ solid-water interfacial energy.

$S^{\prime}{ }_{12}=$ Solid-water interfacial energy $+S_{32}\{(1-x)(1-\sigma)\}+$ solid-organic/liquid interfacial energy.

$$
\therefore \quad \cos \theta_{32}=\frac{S_{13}^{\prime}-S^{\prime}{ }_{12}}{S_{32}}=2 \sigma-1+2 x(1-\sigma)
$$

Hence if $x$ increases, $\theta_{32}$ diminishes.

(ii) If $\theta_{32}$ is obtuse, the craters should be practically free from water.

$\therefore \quad S^{\prime}{ }_{13}=S_{32} \sigma(1-x)+$ solid-liquid interfacial energy + solid-water interfacial energy $S^{\prime}{ }_{12}=S_{32}\{x+(1-\sigma)(1-x)\}+$ solid-liquid interfacial energy + solid-water interfacial energy

$\therefore \quad \cos \theta_{32}=2 \sigma-1-2 \sigma x$

Hence, if $x$ increases, $\theta_{32}$ will increase.

The broad conclusions of the above theoretical considerations may be summarized as follows :

(i) $\theta_{32}$ decreases with increase in the hydrophilic property of the metal surface;

(ii) $\theta_{32}$ diminishes as roughness increases if it is acute, and increases with roughness if it is obtuse.

On the basis of the above theory, the results of the present study of aluminum surfaces can be interpreted. There are two distinct cases in the preferential wetting property of aluminum surfaces: (i) where the metal surface is preferentially wetted by water, i. e., $\theta_{32}$ is small; (ii) where the metal surface is preferentially wetted by oil, i. e., $\theta_{32}$ is large. These are illustrated in figures 3 (a) and 3 (b).

Let us examine case (i) in detail :

Polished aluminum (mechanically as well as electrolytically) exhibits an obtuse angle of contact $\left(\theta_{32}\right)$ which shows that it is preferentially wetted by oil. This is perhaps mainly due to a film of impurities adsorbed by the smooth aluminum surface with the hydrophobic groups orienting themselves outwards. The higher contact angle exhibited when oleic acid is added to liquid paraffin can be explained by assuming that the oxidized aluminum surface reacts with the fatty acid, to form a layer of the salt, aluminum oleate, the hydrocarbon chains of which are projected outwards. The hydrocarbon chains of the oleate layer are not easily wetted by water and hence the contact angle $\left(\theta_{32}\right)$ increases.

Case (ii) : All types of anodized aluminum (example, in $\mathrm{H}_{2} \mathrm{SO}_{4}, \mathrm{H}_{2} \mathrm{Cr}_{2} \mathrm{O}_{7}$ and $\mathrm{H}_{3} \mathrm{PO}_{4}$ ) and etched aluminum (in $20 \% \mathrm{HCl}$ with $0.1 \% \mathrm{CuCl}_{2}$ ) exhibit an acute angle of contact $\left(\theta_{32}\right)$ showing its preferential wettability by water. This is due to the hydroxyl groups of aluminum hydroxide having a high 
attraction towards water. The anodic oxide films are highly porous and their porosity depends upon the electrolyte used. Because of large adsorbing surface in the porous film, the surface active impurities cannot cover any major portion of the surface and hence the wetting properties are not appreciably altered. The differences noted in the values of $\theta_{32}$ for plates anodized in different electrolytes is presumably due to the differences in roughness. It is known that the porosity of the oxide film differs with plates anodized in different baths. In the following table ${ }^{11}$ the values of pore diameter are given for the anodized plates in different electrolytes.

Table 2 Values of Pore Diameter for the Plates Anodized in Different Electrolytes

\begin{tabular}{c|c|c|c|c|c}
\hline \hline S. No. & Electrolyte & $\begin{array}{c}\text { Concentration } \\
\text { (\% wt.) }\end{array}$ & Temp. $\left({ }^{\circ} \mathrm{F}\right)$ & Pore dia. $(\AA)$ & $\theta_{32^{*}}$ \\
\hline 1 & $\mathrm{H}_{3} \mathrm{PO}_{4}$ & 4 & 75 & 330 & $27^{\circ}$ \\
2 & $\mathrm{H}_{2} \mathrm{Cr}_{2} \mathrm{O}_{7}$ & 3 & 100 & 240 & $39^{\circ}$ \\
3 & $\mathrm{H}_{2} \mathrm{SO}_{4}$ & 15 & 50 & 120 & $54^{\circ}$ \\
\hline
\end{tabular}

* These are the experimental values obtained by the authors.

From the above theoretical considerations, it is clear that $\theta_{32}$ values can be used to judge the roughness of a surface. With anodized plate, since the $\theta_{32}$ value is acute, the roughness should be higheer as $\theta_{32}$ values get lower. This can be made use of for interpreting the available data as given in Table 2 , the order of pore diameters which gives an idea of the roughness happens to be the reverse of the order of $\theta_{32}$ values. It is also possible that the different $\theta_{32}$ values may be the result of intrinsic differences of wettability. Regarding the etched plates it is difficult to make any comment as the roughness would depend upon the exact method of etching and the contact angle would depend on the etchpattern.

\section{Acknowledgement}

The authors' thanks are due to Prof. K.S.G. Doss, Director, Central Electrochemical Research Institute, Karaikudi for having suggested the problem and for his valuable discussions. Our thanks are also due to Shri S.K. Rangarajan for his helpful suggestions during the course of this work.

(Received Apr. 20, 1962)

\section{Literature :}

1) Richards, Carver, J. Am. Chem. Soc. 43, 827 (1921).

2) N.K. Adam, G. Jessop, J. Chem. Soc. 1865 (1925).

3) G.L. Mack, J. Phys. Chem. 158 (1936).

4) R.A.C. Adams, International Bulletin (Jan. 1956).

5) K.S.G. Doss, M.Sc. Thesis, Mysore University (1933).

6) K.S.G. Doss, B.S. Rao, Proc. Indi. Acad. Sci. 7 A, 113 (1938).

7) H.K. Livingston, J. Phys. Chem. 48, 120 (1944).

8) T. Young, Phil. Trans. 54 (1805).

9) Harkins, Dahlstrom, Ind. Eng. Chem. 22, 900 (1930).

10) I. Langmuir, Chem. Rev. 6, 467 (1929).

11) Wernick, Pinner, "Surface Treatment of Aluminium and Its Alloys" pp. 230 (1956), Robert Draper Ltd., Middlesex. 